\title{
Anomalous swelling of multilamellar lipid bilayers in the transition region by renormalization of curvature elasticity
}

Callisen, Thomas Hønger; Mortensen, Kell; Ipsen, John Hjorth; Lemmich, Jesper; Bauer, Rogert; Mouritsen, Ole G.

Published in:

Physical Review Letters

Link to article, DOI:

10.1103/PhysRevLett.72.3911

Publication date:

1994

Document Version

Publisher's PDF, also known as Version of record

Link back to DTU Orbit

Citation $(A P A)$ :

Callisen, T. H., Mortensen, K., Ipsen, J. H., Lemmich, J., Bauer, R., \& Mouritsen, O. G. (1994). Anomalous swelling of multilamellar lipid bilayers in the transition region by renormalization of curvature elasticity. Physical Review Letters, 72(24), 3911-3914. https://doi.org/10.1103/PhysRevLett.72.3911

\section{General rights}

Copyright and moral rights for the publications made accessible in the public portal are retained by the authors and/or other copyright owners and it is a condition of accessing publications that users recognise and abide by the legal requirements associated with these rights.

- Users may download and print one copy of any publication from the public portal for the purpose of private study or research.

- You may not further distribute the material or use it for any profit-making activity or commercial gain

- You may freely distribute the URL identifying the publication in the public portal 


\title{
Anomalous Swelling of Multilamellar Lipid Bilayers in the Transition Region by Renormalization of Curvature Elasticity
}

\author{
Thomas Hønger, ${ }^{1}$ Kell Mortensen, ${ }^{2}$ John Hjort Ipsen, ${ }^{1}$ Jesper Lemmich, ${ }^{1}$ Rogert Bauer, ${ }^{3}$ and Ole G. Mouritsen ${ }^{1}$ \\ ${ }^{1}$ Department of Physical Chemistry, The Technical University of Denmark, Building 206, DK-2800 Lyngby, Denmark \\ ${ }^{2}$ Department of Solid State Physics, Risø National Laboratory, DK-4000 Roskilde, Denmark \\ ${ }^{3}$ Department of Physics, Royal Danish School of Agricultural and Veterinary Sciences, Thorvaldsensvej 40 , \\ DK-1871 Frederiksberg, Denmark
}

(Received 8 September 1993)

\begin{abstract}
Small-angle neutron scattering is used to determine the temperature dependence of the lamellar repeat distance in an aqueous multilamellar solution of phospholipid bilayers. A thermal anomaly in the swelling behavior is observed at the bilayer phase transition. The anomalous behavior can be suppressed by varying the lipid acyl-chain length or by alloying with a molecular stiffening agent. The experimental results are explained in terms of renormalization of the bilayer curvature elasticity and by using a theory of repulsive interlamellar undulation forces.
\end{abstract}

PACS numbers: $87.64 . \mathrm{Bx}$

Lipid bilayers, which are models of biological membranes [1], exhibit a richness of structures and phase equilibria due to an intricate interplay between different intrabilayer and interbilayer forces. These forces, which often compete and are of very different origin and operate on different length scales, include van der Waals forces, electrostatic double-layer forces, hydration forces, surface dipole-dipole forces, as well as steric forces [2]. In aqueous solution, three-dimensionally structured lipid phases may be formed as multilamellar stacks of bilayers, each having the property of a two-dimensional smectic liquid crystal [3]. The lipid bilayer has negligible surface tension. Whereas an isolated single bilayer due to its extreme softness exhibits large thermally induced undulations, these undulations are considerably suppressed in a lamellar stack. This originally led Helfrich [4] to advance the idea of a repulsive undulation force acting between the bilayers. The undulation force, which is important for controlling the swelling behavior of the lamellar stack, is a long-range force generated by the out-ofplane bilayer fluctuations and it is therefore of steric and entropic origin. Helfrich [4] showed by simple scaling arguments that the free-energy density, $f_{u}$, which leads to undulation forces for fluid membranes, takes the form

$$
f_{u}=(3 \pi / 128)\left(k_{B} T\right)^{2} / \kappa d^{2},
$$

where $d$ is the lamellar repeat distance and $\kappa$ is the elastic bending modulus for a single bilayer (the curvature elasticity) which is a material constant. For sufficiently soft bilayers the undulation force plays an important role for the swelling behavior.

There are different ways of manipulating the bilayer system in order to expose the manifestations of the repulsive undulation force [5-14], e.g., by varying the composition of the aqueous solution in order to shift the balance between the different competing interlamellar forces. Another way is to change the lipid material itself or to incorporate into the bilayer various solutes which change the curvature elasticity $[12,15]$. Yet another way is to study the swelling properties for a given lipid material in the region around the main phase transition temperature, $T_{m}$, where the softness of the bilayer exhibits a thermal anomaly. The main transition is a kind of firstorder solid-liquid phase transition [16] which is close to a critical point, $T^{*}$ (pseudocritical) $[16,17]$. At the main transition, the bilayer exhibits large density fluctuations which are reflected in thermal anomalies in the specific heat as well as the lateral area compressibility $[16,18]$.

In this Letter we present the results of the first experimental study which directly demonstrates the appearance of anomalous swelling behavior in a multilamellar phospholipid bilayer system in the immediate vicinity of the phase transition. The results clearly demonstrate the effects of the undulation forces. The data are obtained by small-angle neutron-scattering (SANS) techniques. Using a modified version of the Helfrich theory [19] of membrane conformations we interpret this anomalous behavior in terms of a fluctuation-induced renormalization of the curvature elasticity. The theory accounts for the coupling between the curvature and the density fluctuations of the bilayer. We present experimental data which show how the anomalous swelling behavior can be suppressed by choosing a lipid material which forms bilayers whose phase transition is further removed from the critical point and hence sustain less dramatic density fluctuations. It is furthermore indicated how incorporation of various solutes into the bilayer can change the swelling behavior via a change in the curvature elasticity.

Two different bilayer-forming lipid materials were used in the present study, dimyristoyl phosphatidylcholine (DMPC) and dipalmitoyl phosphatidylcholine (DPPC) which have saturated fatty-acid chain lengths corresponding to 14 and 16 carbon atoms, respectively. The lipids with per-deuterated fatty acid chains, DMPC- $d_{54}$ and DPPC- $d_{62}$, were purchased from Avanti Polar Lipids (Birmingham, AL), and used without further purification. The multilamellar bilayers were formed by dissolving the dry lipid in a $\mathrm{D}_{2} \mathrm{O}$ buffer solution of $50 \mathrm{mM}$ Hepes 
and $10 \mathrm{mM} \mathrm{NaCl}$ at $p \mathrm{H} \mathrm{7.6.} \mathrm{The} \mathrm{sample} \mathrm{condition} \mathrm{is} \mathrm{that}$ of excess water.

The samples were mounted in sealed quartz containers (Suprasil from Hellma, FRG) with $2 \mathrm{~mm}$ flight path. The neutron beam at the sample position was $7 \mathrm{~mm}$ in diameter. For the neutron spectra of water, used for calibration, a $1 \mathrm{~mm}$ thick quartz container was used. The smallangle neutron scattering experiments were performed using the Ris $\varnothing$-SANS facility. The results shown below were obtained, in the case of the DMPC samples, using $3.1 \AA$ neutrons with a sample-to-detector distance of $1.5 \mathrm{~m}$, giving scattering vectors $q=(4 \pi / \lambda) \sin (\theta / 2)$ in the range $0.04-0.35 \AA^{-1}$, where $\theta$ is the scattering angle and $\lambda$ is the neutron wavelength. The DPPC samples were measured using $3.5 \AA$ neutrons with a sample-to-detector distance of $3 \mathrm{~m}$, giving scattering vectors $q$ in the range $0.02-0.2 \AA^{-1}$. The neutron wavelength resolution was $\Delta \lambda / \lambda=0.18$, and the neutron beam collimation was determined by the pinhole sizes of $16 \mathrm{~mm}$ and $7 \mathrm{~mm}$ diameter at source and sample position, respectively. The smearing induced by the different instrumental procedures is taken into account in the data analysis [20]. The scattering data were corrected for background arising from the quartz container with $\mathrm{D}_{2} \mathrm{O}$ and from other sources, as measured with the neutron beam blocked by plastic-containing boron at the sample position. The incoherent scattering from $\mathrm{H}_{2} \mathrm{O}$ was used to take the deviation from a uniform detector response into account. The scattering patterns were all azimuthally isotropic. The data have therefore been reduced to the one-dimensional $I(q)$ scattering functions only dependent on the absolute value of the wave vector.

The neutron scattering patterns are dominated by the (10) Bragg reflection which signals the bilayer periodicity in the multilamellar stack. For the DPPC sample, the spectrometer settings were chosen to allow better resolution of the (10) peak, with the cost that higher-order reflections are not included in the $q$ ranges covered. (In the scattering function for DPPC, an additional structure appears at $q \sim 0.04 \AA^{-1}$ in the temperature range $T_{p} \simeq 32^{\circ} \mathrm{C}$ to $T_{m} \simeq 39^{\circ} \mathrm{C}$, corresponding to the ripple structure of the $P_{\beta^{\prime}}$ phase.) The lowest-order reflection shows that the typical lamellar periodicity corresponds to a wave vector of the order of $0.1 \AA^{-1}$. The (10) reflection was analyzed using a least-squares fitting routine to a Lorentzian line shape, $I(q)=I_{0} /\left[1+\left(q-q_{0}\right)^{2} \ell^{2}\right]+I_{1}$, where $\ell$ is the correlation length, $I_{0}$ is the prefactor, and $I_{1}$ denotes the incoherent background arising from the sample.

The resulting Bragg peak at $q_{0}$, corresponding to the lamellar periodicity, shows a substantial temperature dependence demonstrating that there is an overall decrease in the repeat distance, $d=2 \pi / q_{0}$, by going from the solid phase to the liquid phase. Figure 1 shows on a reduced temperature scale the resulting repeat distance for both DMPC and DPPC. These results are consistent with earlier observations using both x-ray $[21,22]$ and neutron scattering $[23,24]$.
The most striking observation made from the data set is, however, the dramatic nonmonotonic variation of the periodicity of the DMPC sample close to the phase transition. The enhanced lamellar repeat distance at the transition indicates an anomalous behavior with pronounced swelling close to the phase transition of DMPC. In the case of DPPC, the neutron scattering data presented in Fig. 1 indicate a small anomaly which, however, can be barely discerned. The finding of this anomalous swelling behavior of phospholipid bilayers in their phase transition region, which seems not to have been noted in earlier scattering studies, constitutes the main experimental result of the present work.

During the transition the thickness of the bilayer decreases [21] which is the main source to the overall decrease in lamellar repeat distance seen in Fig. 1. However, a full analysis [25] of the experimental scattering function in terms of a model that allows for independent variation of the thicknesses of the different layers shows that it is an anomalous increase in the water layer thickness which is mainly responsible for the peak in the bilayer periodicity seen in Fig. 1. It should be pointed out that our result for the lamellar periodicity presented in Fig. 1 does not depend on any specific model assumption.

The experimental finding of anomalous swelling behavior of multilamellar phospholipid bilayers in the transition region can be rationalized theoretically via the effective steric interaction, Eq. (1), induced by an excluded volume effect and thermal membrane undulations in combination with a thermal renormalization of the elastic bending modulus by lateral density fluctuations in the phase transition region. This effect will be briefly described. First we consider the bending rigidity of a single bilayer in the phase transition region and then we use Eq. (1) to derive the undulation force between layers in a lamellar stack. We restrict ourselves in the theoreti-

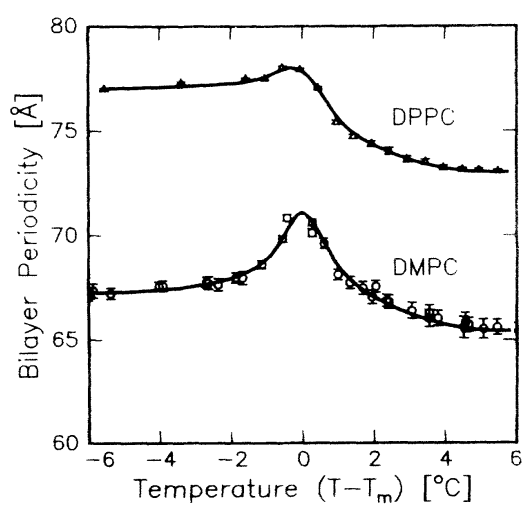

FIG. 1. Lamellar repeat distance, $d(T)$, for multilamellar bilayers of DMPC- $d_{54}$ and DPPC- $d_{62}$ plotted as functions of reduced temperature, $T-T_{m}$, where $T_{m}$ is the main transition temperature $\left(T_{m}=17.3^{\circ} \mathrm{C}\right.$ and $39.0^{\circ} \mathrm{C}$ for DMPC- $d_{54}$ and DPPC- $d_{62}$, respectively). The data are derived from Lorentzian fits to the neutron-scattering data. The solid lines are guides to the eye. 
cal description to the liquid side of the phase transition region, which is strongly dominated by lateral density fluctuations caused by fluctuations in acyl-chain conformational degrees of freedom [26]. Hence, we need not be concerned with specific differences between the two phases, such as the fact that the solid phase has a finite shear rigidity [1]. Nevertheless, we expect that a thermal renormalization effect on the bending elasticity will also be present on the solid side of the transition.
The local, lateral density fluctuations in the two monolayer halves of a single bilayer is described by two Gaussian fluctuating scalar fields, $\phi_{1}$ and $\phi_{2}$. These fluctuating fields, which describe the lateral cooperativity in acylchain conformational degrees of freedom, become very soft in the phase transition region $[18,26]$. In terms of the local density difference, $\psi=\frac{1}{2}\left(\phi_{1}-\phi_{2}\right)$, between the monolayers, the free-energy functional of nearly flat bilayer membrane conformations, $(x, y, z(x, y))$, can be written as

$$
\mathcal{H}[z(\mathbf{x}), \psi(\mathbf{x})]=\kappa_{0} \int d^{2} \mathbf{x}\left\{\frac{1}{2}[\Delta z(\mathbf{x})]^{2}+\frac{1}{2}\left[c(\nabla \psi(\mathbf{x}))^{2}+K \psi(\mathbf{x})^{2}\right]+r \psi(\mathbf{x}) \Delta z(\mathbf{x})\right\}
$$

where $\mathbf{x}=(x, y)$. The first term in Eq. (2) is a linerized version of Helfrich's general free energy for fluid membrane conformations [19] which forms the basis for the description of small out-of-plane membrane configurations in a lamellar system leading, e.g., to Eq. (1). $\kappa_{0}$ is the bare membrane bending elasticity modulus. The second term represents a simple harmonic description of the inhomogenous density-difference fluctuations. $K^{-1}$ is proportional to the lateral compressibility modulus which is strongly increased in the transition region [18]. The stiffness coefficient, $c$, determines together with $K$ a characteristic length scale, $\xi=\sqrt{c / K}$, for the lateral density fluctuations. In a first approximation, the local density difference, $\psi$, couples linearly to the local mean curvature $(\sim \Delta z)$, as expressed in the last term, and the associated phenomenological coupling constant, $r$, has the dimension of inverse length. The parameters $c, K$, and $r$ are chosen with respect to the energy unit $\kappa_{0}$. The coupling between mean curvature and nonhomogeneous density variations has recently been considered theoretically in the description of the intermonolayer friction of lipid bilayers [27] and the interplay between composition and conformation in two-component vesicles $[28,29]$.

The $\psi$ field can now be integrated out in Eq. (2) leading to

$$
\mathcal{H}[z(\mathbf{x})]=\frac{\kappa_{0}}{2} \int d^{2} \mathbf{x}[\Delta z(\mathbf{x})]^{2}-\frac{\kappa_{0} r^{2}}{2} \int d^{2} \mathbf{x}_{1} \int d^{2} \mathbf{x}_{2} \Delta z\left(\mathbf{x}_{1}\right) G\left(\mathbf{x}_{1}, \mathbf{x}_{2}\right) \Delta z\left(\mathbf{x}_{2}\right)+\text { const }
$$

where $G$ is the usual Ornstein-Zernike corellator,

$$
G\left(\mathbf{x}_{1}, \mathbf{x}_{2}\right)=\int \frac{d^{2} \mathbf{k}}{(2 \pi)^{2}} \frac{e^{i \mathbf{k} \cdot\left(\mathbf{x}_{1}-\mathbf{x}_{2}\right)}}{c \mathbf{k}^{2}+K}
$$

In order to extract the effective free energy governing the conformational behavior of the membrane at distances larger than $\xi$, a simple derivative expansion of $G$ can be applied in Eq. (3):

$$
\begin{aligned}
\mathcal{H}[z(\mathbf{x})]= & \frac{\kappa_{0}}{2}\left(1-\frac{r^{2}}{K}\right) \int d^{2} \mathbf{x}[\Delta z(\mathbf{x})]^{2} \\
& + \text { higher derivatives of } z+\text { const. }
\end{aligned}
$$

Equation (5) shows that the bending modulus is effectively reduced to

$$
\kappa=\kappa_{0}\left(1-r^{2} \xi^{2} / c\right),
$$

where we have used $\xi=\sqrt{c / K}$. Order-of-magnitude considerations for the parameter values in the transition region $[26,28]$ lead to $c \sim 1, r \sim 0.1 \mathrm{~nm}^{-1}$, and $\xi \sim 1-10 \mathrm{~nm}$, which implies that one should expect a considerable reduction of the bending modules in the transition region for $T>T_{m}$. This expectation is indeed supported by recent experimental measurements of $\kappa$ in the transition region of DMPC and DPPC [30]. A result similar to Eq. (6) has been derived in the context of two-component membranes [29].
By combining the result in Eq. (6) for a single bilayer with the simplified expression for Helfrich's free-energy density, Eq. (1), for sterically interacting bilayers, we obtain the main qualitative result of our calculation that there is an enhanced interlamellar repulsion in the transition region and the lamellar repeat distance will consequently blow up. A more refined theoretical description must take into account the details and renormalization of the intermembrane potential $[8,13]$. Furthermore, a detailed description in the low-temperature phase must take the lateral ordering (and hence the finite shear rigidity) into account. Nevertheless, due to the extreme softening of the lipid bilayer in the transition region [18] the main part of the anomalous swelling effect is accounted for by Eq. (6).

The various material parameters entering the expression for the renormalized curvature elasticity modulus in Eq. (6) are not known with sufficient accuracy to allow us to make quantitative predictions. Nevertheless, it is known from micromechanical experiments that $\kappa_{0}$ is smaller for DMPC than for DPPC [15] and from NMR experiments and model calculations that the phase transition in DMPC is closer to a critical point than in DPPC $[17,26]$. Using Eq. (6), this implies that the renormalized curvature elasticity modulus $\kappa$ is smaller for DMPC 
than for DPPC. Hence the prediction from Eq. (1) is that the anomalous swelling at the phase transition for DMPC should be more pronounced than for DPPC. This prediction is in good agreement with the results of the neutron-scattering experiments in Fig. 1.

We wish to put the results of the present work into a broader context by pointing out that a rich variety of amphiphilic molecular compounds exist which can be intercalated into lipid bilayers and form a kind of lipid alloy system. It is likely that any conceivable mechanical property, including curvature elasticity, can be modulated in a very wide range by choosing an appropriate alloying compound, either a synthetic one or one provided by nature to serve a specific purpose in a biological membrane $[29,31]$. We have found from SANS measurements [25] of the type described in the present Letter that incorporation of small amounts of a molecular stiffening agent, such as the transmembrane channelformer gramicidin A, into DMPC bilayers leads to a suppression of the anomalous swelling behavior, consistent with the expectation that gramicidin A increases the bilayer bending rigidity. A prominent example of a natural alloying species for lipid bilayers is cholesterol, which nature has evolved for membrane purposes to provide high mechanical coherence and low membrane permeability. Another example involves certain bipolar lipids which have the opposite effect of cholesterol by lowering the curvature elasticity modulus leading to hyperelastic bilayers which are so soft that they behave almost like a completely flexible surface [12].

In summary, we have provided experimental evidence from SANS measurements on multilamellar phospholipid bilayer systems which show anomalous swelling behavior in the phase transition region. The anomaly was explained in terms of a thermally renormalized curvature elasticity modulus by using a modified version of Helfrich's model for membrane conformations [19] which incorporates an instantaneous spontaneous curvature that is induced by the difference in density in the two monolayers of the bilayer. The resulting reduced bending modulus gives rise to increased membrane flexibility and entropy-driven swelling of the lamellar lipid-bilayer structure, cf. Eq. (1). The swelling anomaly can be suppressed by either choosing a lipid material whose phase transition is further removed from criticality or by incorporating a molecular stiffening agent into the bilayer, thereby increasing the curvature elasticity.

This work was supported by the Danish Natural Science Research Council under Grants No. 11-0065-1, No. 11-8370, and No. 11-9651..O.G.M. is an Associate Fellow of the Canadian Institute of Advanced Research.
[1] M. Bloom, E. Evans, and O. G. Mouritsen, Quart. Rev. Biophys. 24, 293 (1991).

[2] J. Israelachvili, Intermolecular and Surface Forces (Academic Press, New York, 1991), p. 450.

[3] C. Gebhardt, H. Gruler, and E. Sackmann, Z. Naturforsch. 32c, 581 (1977).

[4] W. Helfrich, Z. Naturforsch. 33a, 305 (1978).

[5] C. R. Safinya, D. Roux, G. S. Smith, S. K. Sinha, P. Dimon, N. A. Clark, and A. M. Bellocq, Phys. Rev. Lett. 57, 2718 (1986).

[6] F. C. Larche, J. Appell, G. Porte, P. Bassereau, and J. Marignan, Phys. Rev. Lett. 56, 1700 (1986).

[7] E. Evans and V. A. Parsegian, Proc. Natl. Acad. Sci. (USA) 83, 7132 (1986).

[8] S. Leibler and R. Lipowsky, Phys. Rev. B 35, 7004 (1987).

[9] D. Roux and C. R. Safinya, J. Phys. (Paris) 49, 307 (1988)

[10] G. Gompper and D. M. Kroll, Europhys. Lett. 9, 59 (1989).

[11] S. Leibler and A. C. Maggs, Phys. Rev. Lett. 63, 406 (1989).

[12] E. Sackmann, Can. J. Phys. 68, 999 (1990).

[13] E. Evans and J. H. Ipsen, Electrochim. Acta 36, 1735 (1991).

[14] W. Helfrich, J. Phys. II (France) 3, 385 (1993).

[15] E. Evans and D. Needham, J. Phys. Chem. 91, 4219 (1987).

[16] O. G. Mouritsen, Chem. Phys. Lipids 57, 178 (1991).

[17] M. R. Morrow, J. P. Whitehead, and D. Lu, Biophys. J. 63, 18 (1992).

[18] E. Evans and R. Kwok, Biochemistry 21, 4874 (1982).

[19] W. Helfrich, Z. Naturforsch. 28c, 693 (1973).

[20] J. S. Pedersen, D. Posselt, and K. Mortensen, J. Appl. Cryst. 23, 321-333 (1990).

[21] Y. Inoko and T. Mitsui, J. Phys. Jpn. 44, 1918 (1978).

[22] M. J. Janiak, D. M. Small, and G. G. Shipley, J. Biol. Chem. 254, 6068 (1979).

[23] G. Büldt, H. U. Gally, J. Seelig, and G. Zaccai, J. Mol. Biol. 134, 673 (1979).

[24] M. C. Wiener and S. H. White, Biophys. J. 59, 162 (1991).

[25] T. Hønger, K. Mortensen, J. H. Ipsen, J. Lemmich, R. Bauer, and O. G. Mouritsen (unpublished).

[26] J. H. Ipsen, K. Jørgensen, and O. G. Mouritsen, Biophys. J. 58, 1099 (1990).

[27] U. Seifert and S. A. Langer, Europhys. Lett. 23, 71 (1993).

[28] U. Seifert, Phys. Rev. Lett. 70, 1335 (1993)

[29] S. Leibler, J. Phys. (Paris) 47, 507 (1986).

[30] L. Fernandez-Puente, I. Bivas, M. D. Mitov, and P. Méléard (to be published).

[31] M. Bloom and O. G. Mouritsen, in "Handbook on Membranes I: Structure and Conformation," edited by R. Lipowsky and E. Sackmann (to be published). 